\title{
Optimization Of Mechanical Strength Of Rotary-Vane Engine
}

\author{
Yury Zhuravlev, Andrey Perminov, Yury Lukyanov, Sergey Tikhonov, \\ Alexander Ilyin, Sergey Semyonov, \\ Pskov State University, Electromechanical Faculty, \\ Department of Electric Drive and Automation Systems. \\ Address: Lenina 8, Pskov, 180000, Russia
}

\begin{abstract}
The article discusses a rotory-vane heat engine with a lever-cam mechanism motion conversion (an engine may be an internal combustion or external combustion). The output shaft of the engine adds drive torque from the working fluid pressure forces acting on the blade and the inertial moment of the forces of inertia of engine components. The mechanical strength of the motor is dependent on the magnitude and phase of these two torque. The purpose of the article is to determine the conditions under which mechanical strength is minimized.
\end{abstract}

Keywords: rotary-vane engine, lever-cam motion converter, external supply of heat.

\section{INTRODUCTION}

It is known that internal combustion engines have almost reached the limit of their energy and environmental characteristics. Therefore, it is necessary to develop engines of a new generation, constructed using other design schemes and implementing new thermodynamic cycles. To solve this problem rotor-vane machines with external heat supply can be used.

The Fig. 1 shows the engine component diagram.

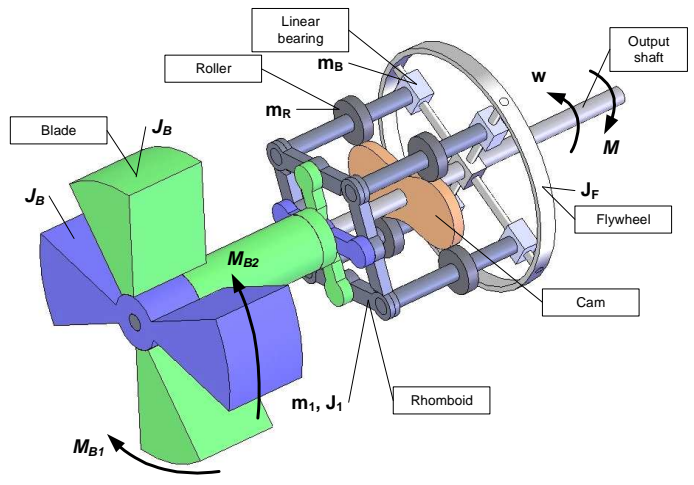

Fig. 1. Engine component diagram.

The engine consists of a driving part, motion converter, output shaft to the flywheel. The driving part consists of two coaxial rotors. Each rotor has the blade of the two diametrically opposite vanes (pistons). Also, the rotors have forks with two diametrically arranged fingers. The fingers are pivotally connected to the midpoints of the sides of the rhomboid links. The pressure of the working fluid between two adjacent vanes create moments on the blades $M_{B 1}=-M_{B 2}$, which depend on the output shaft angle.

The tops rhomboid are rollers. The rollers contact with a stationary profiled cam surface. Rhomboid is a motion transformer. It converts cyclic motion of the blade (within the angles $\psi_{\text {min }}, \psi_{\text {max }}$ between them) into a unidirectional rotation of the output shaft. Thus transmitted torque from the blades. Torque transmission from rhomboid to the output shaft occurs via linear bearings.

To the output shaft of the engine is attached a load torque $M$.

The motion transformer uses a cam profile which in polar coordinates $\rho, \alpha$ is given by [1]:

$$
\rho(\alpha)=2 l \sin (a+b \sin 2 \alpha)
$$

where $2 l-$ rhomboid link length, $a=\pi / 4$; $b=\pi / 4-\psi_{\min } / 2$, where $\psi_{\min }-$ angle between the axes of the blades (usually $\psi_{\min }=50^{\circ}-55^{\circ}$ ).

The vane angles change in a law:

$$
\begin{gathered}
\varphi_{1}(\alpha)=\frac{3 \pi}{4}+\alpha+b \cos 2 \alpha, \\
\varphi_{2}(\alpha)=\frac{\pi}{4}+\alpha-b \cos 2 \alpha .
\end{gathered}
$$

The dynamic system has one degree of freedom, because position of all elements is determined by one coordinate - output shaft angle $\alpha$.

The motion equation of the system can be written in the form of Lagrange second kind equation: 


$$
\frac{d}{d t} \frac{\partial T}{\partial \dot{\alpha}}-\frac{\partial T}{\partial \alpha}=M_{D T}-M
$$

where $T$ - the kinetic energy of the system; $M_{D T}-$ the drive torque at the output shaft; $M$ - the load torque.

Equation (3) can also be written as an kinetostatic equilibrium equation:

$$
L_{Z}+M_{D T}-M=0
$$

where $L_{Z}-$ motor torque inertia forces applied to the output shaft:

$$
L_{Z}=-\left(\frac{d}{d t} \frac{\partial T}{\partial \dot{\alpha}}-\frac{\partial T}{\partial \alpha}\right)
$$

\section{TORQUE OF INERTIA FORCES}

The kinetic energy of the system includes the kinetic energy of the two blades $T_{B}$, four links of the rhomboid $T_{L}$, four rollers rhomboid $T_{R}$, four linear bearings with fingers $T_{L B}$, output shaft with a flywheel $T_{F}$ :

$$
T=T_{B}+T_{L}+T_{R}+T_{L B}+T_{F} .
$$

Rotors carrying blades, make a rotary motion around a fixed axis with angular velocity $\dot{\varphi}_{1}, \dot{\varphi}_{2}$. Kinetic energy of rotors carrying blades $T_{B}=J_{B}\left(\dot{\varphi}_{1}^{2}+\dot{\varphi}_{2}^{2}\right) / 2, \quad$ where $\quad J_{B}=J_{B 1}=J_{B 2} \quad-$ moments of inertia of the blades B1 and B2; $\dot{\varphi}_{1}=\dot{\alpha}(1-2 b \cdot \sin 2 \alpha) ; \quad \dot{\varphi}_{2}=\dot{\alpha}(1+2 b \cdot \sin 2 \alpha)$.

$$
T_{B}=\frac{1}{2} \dot{\alpha}^{2} 2 J_{B}\left(1+2 b^{2}-2 b^{2} \cos 4 \alpha\right)
$$

Links rhomboid make plane motion, so their kinetic energy is the sum of the kinetic energies of the translational and rotational motions: $T_{L}=\left[m_{1}\left(2 V_{C 1}^{2}+2 V_{C 2}^{2}\right)+J_{1}\left(2 \Omega_{1}^{2}+2 \Omega_{2}^{2}\right)\right] / 2, \quad$ where $m_{1}, J_{1}$ - mass and moment of inertia about the central axis of one link; $V_{C 1}, V_{C 2}$ - linear velocities of the centers; $\Omega_{1}, \Omega_{2}-$ angular velocities of the centers links 1 and 2 . Given that

$$
\begin{aligned}
& V_{C 1}^{2}+V_{C 2}^{2}=\dot{\alpha}^{2} l^{2}\left(2+4 b^{2}-4 b^{2} \cos 4 \alpha\right), \\
& \Omega_{1}^{2}+\Omega_{2}^{2}=\dot{\alpha}^{2}\left(2+4 b^{2}-4 b^{2} \cos 4 \alpha\right) \\
& T_{L}=\frac{1}{2} \dot{\alpha}^{2} 4\left[\left(m_{1} l^{2}+J_{1}\right)\left(1+2 b^{2}-2 b^{2} \cos 4 \alpha\right)\right] .
\end{aligned}
$$

Four roller rhomboid has kinetic energy

$$
T_{R}=\frac{1}{2} \dot{\alpha}^{2} 8 m_{R} l^{2}\left(1+2 b^{2}-2 b^{2} \cos 4 \alpha\right),
$$

where $m_{R}$ - mass of one roller.

The kinetic energy of the four linear bearings include of the kinetic energy of rotational motion $T_{r o t}=\frac{1}{2} \dot{\alpha}^{2} 4 J_{L B}$ and kinetic energy of translational motion $T_{t r}=\frac{1}{2} 2 m_{L B}\left(V_{A}^{2}+V_{B}^{2}\right)$, where $V_{A}, V_{B}-$ absolute tops speed rhomboid $\mathrm{A}$ and $\mathrm{B} ; m_{L B}, J_{L B}-$ mass and moment of inertia of linear bearings. We obtain

$$
\begin{gathered}
T_{L B}=\frac{1}{2} \dot{\alpha}^{2} . \\
\cdot\left[4 J_{L B}+8 m_{L B} l^{2}\left(1+2 b^{2}-2 b^{2} \cos 4 \alpha\right)\right] .
\end{gathered}
$$

Flywheel together with the output shaft has a kinetic energy:

$$
T_{F}=\frac{1}{2} \dot{\alpha}^{2} J_{F}
$$

where $J_{F}-$ moment of inertia of flywheel together with the output shaft.

Summing up the values of the kinetic energy (7) (11), we obtain the kinetic energy of the engine:

$$
T=\frac{1}{2} \dot{\alpha}^{2} J(\alpha),
$$

where $J(\alpha)$ - moment of inertia of engine referred to the output shaft. Moment of inertia includes fixed and variable components

$$
J(\alpha)=J_{C}-J_{V} \cos 4 \alpha .
$$

There are $J_{C}-$ permanent component; $J_{V}-$ amplitude of the harmonic component with a period of $\pi / 2$ :

$$
\begin{gathered}
J_{C}=J_{F}+4 J_{L B}+2\left(1+2 b^{2}\right) . \\
\cdot\left[J_{B}+2\left(m_{1} l^{2}+J_{1}\right)+4 l^{2}\left(m_{R}+m_{L B}\right)\right] ; \\
J_{V}=4 b^{2}\left[J_{B}+2\left(m_{1} l^{2}+J_{1}\right)+4 l^{2}\left(m_{R}+m_{L B}\right)\right] .
\end{gathered}
$$

Expression for the given moment inertia of the engine is:

$$
L_{Z}=-\dot{\alpha}^{2} 2 J_{V} \sin 4 \alpha-\ddot{\alpha}\left(J_{C}-J_{V} \cos 4 \alpha\right) .
$$


Environment. Technology. Resources, Rezekne, Latvia Proceedings of the $11^{\text {th }}$ International Scientific and Practical Conference. Volume III, 357-360

\section{DEFINITION OF DRIVE TORQUE}

Torque of gas pressure forces on the blades $M_{B 1}=-M_{B 2}$ transmitted through the cam motion transformer to the output shaft create a drive torque $M_{D T}$.

Work of drive torque at the elementary move $\delta A\left(M_{D T}\right)=M_{D T} \delta \alpha$ must be equal to work of torques on the vanes $\delta A\left(M_{B 1}, M_{B 2}\right)=M_{B 1} \delta \varphi_{1}+M_{B 2} \delta \varphi_{2}$.

The angles of the vanes $\varphi_{1}$ and $\varphi_{2}$ increment $\delta \varphi_{1}=\frac{d \varphi_{1}}{d \alpha} \delta \alpha$ and $\delta \varphi_{2}=\frac{d \varphi_{2}}{d \alpha} \delta \alpha$, or using (2), $\delta \varphi_{1}=(1-2 b \sin 2 \alpha) \delta \alpha, \quad \delta \varphi_{2}=(1+2 b \sin 2 \alpha) \delta \alpha$.

Moments on the vanes make work

$$
\begin{gathered}
\delta A\left(M_{B 1}, M_{B 2}\right)= \\
{\left[M_{B 1}+M_{B 2}+2 b\left(M_{B 1}-M_{B 2}\right) \sin 2 \alpha\right] \delta \alpha .}
\end{gathered}
$$

Are equal, so the work of the driving torque and torque on the vanes (including $M_{B 2}=-M_{B 1}$ ) we obtain an expression for the type of drive torque

$$
M_{D T}(\alpha)=4 b M_{B 1}(\alpha) \sin 2 \alpha .
$$

It is periodic function of $\alpha$ with period $T=\pi / 2$, which can be expanded in a Fourier series, and represented in the form

$$
\begin{aligned}
& M_{D T}(\alpha)=M_{0}+ \\
& A_{1} \cos 4 \alpha+A_{2} \cos 8 \alpha+A_{3} \cos 12 \alpha+\ldots+ \\
& B_{1} \sin 4 \alpha+B_{2} \sin 8 \alpha+B_{3} \sin 12 \alpha+\ldots
\end{aligned}
$$

where

$$
\begin{gathered}
M_{0}=\frac{1}{T} \int_{0}^{T} M_{D T}(\alpha) d \alpha, \\
A_{k}=\frac{2}{T} \int_{0}^{T} M_{D T}(\alpha) \cos k 4 \alpha d \alpha, \\
B_{k}=\frac{2}{T} \int_{0}^{T} M_{D T}(\alpha) \sin 4 k \alpha d \alpha, \\
(k=1,2, \ldots)
\end{gathered}
$$

$M_{0}$ is the average value of $M_{D T}(\alpha)$ for the period, which is a useful driving torque, which is the product of the angular velocity $\omega=\dot{\alpha}$ equal to engine power $N=M_{0} \omega$. The remaining terms, as a harmonic function of the shaft angle $\alpha=\omega t$, are classified as disturbing moments with frequencies $4 \omega, 8 \omega, 12 \omega, \ldots$ The presence of these harmonics increases the mechanical strength of the engine.

Now we shall find condition under which the mechanical strength of the engine is minimized.

\section{TORQUE ON THE OUTPUT SHAFT}

In the steady state acceleration $\ddot{\alpha}=0$, the angular velocity $\dot{\alpha}=\omega=$ const, and the angle of rotation of the shaft $\alpha=\omega t$.

Inertia moment $L_{Z}$, defined by (5) takes the form $L_{Z}=-\omega^{2} 2 J_{V} \sin 4 \omega t$.

Torque at the output shaft $M_{S}$ equals the sum of the drive torque $M_{D T}$ defined by (18), and the inertial moment $L_{Z}$ :

$$
\begin{gathered}
M_{S}=M_{D T}+L_{Z}= \\
M_{0}+\left(B_{1}-2 J_{V} \omega^{2}\right) \sin 4 \omega t+\tilde{M}(t),
\end{gathered}
$$

where indicated

$$
\begin{gathered}
\tilde{M}(t)=A_{1} \cos 4 \omega t+A_{2} \cos 8 \omega t+\ldots+ \\
B_{1} \sin 4 \omega t+B_{2} \sin 8 \omega t+\ldots
\end{gathered}
$$

When the condition

$$
B_{1}-2 J_{V} \omega^{2}=0
$$

is compensation by inertia forces the most significant component of the disturbing drive torque $B_{1} \sin 4 \omega t$ and, consequently, to minimize the mechanical strength of the engine.

It follows that the design of its engine parameters should be selected so that the nominal angular velocity $\omega_{n}$ meet the condition (20)

$$
\omega_{n}=\omega^{*}=\sqrt{\frac{B_{1}}{2 J_{V}}} .
$$

\section{V.RESULTS OF NUMERICAL SIMULATION}

Initial data for the simulation: torque on output shaft $M_{D T}=M_{0}=1000 \mathrm{~N} \cdot \mathrm{m}$, speed to $1500 \mathrm{rpm}$.

Torques on the blades and the drive torque as functions of the angle $\alpha$ are shown in Fig. 3.

Expansion $M_{D T}(\alpha)$ into a Fourier series (18) gives the following coefficients (in $\mathrm{N} \cdot \mathrm{m}$ ):

$$
\begin{aligned}
& M_{0}=1075, \\
& A_{1}=-217, A_{2}=-468, A_{3}=-266, A_{4}=-72, \\
& B_{1}=1690, B_{2}=478, B_{3}=26, B_{4}=-96 .
\end{aligned}
$$

The most significant component of the disturbing harmonic drive torque is $B_{1} \sin 4 \omega t$, amplitude of which is 1.5 times higher than the constant $M_{0}$. Compensation of this harmonic by inertia forces must take place according to (21) at the angular speed

$$
\omega^{*}=\sqrt{B_{1} / 2 J_{V}}=\sqrt{1690 / 2 * 0,184}=68 c^{-1},
$$

or at a frequency rotation $n^{*}=30 \omega^{*} / \pi=650 \mathrm{rpm}$. 
Inertia moment $L_{Z}=-\omega^{2} 2 J_{V} \sin 4 \omega t$ has an amplitude $A_{L z}=2 J_{V} \omega^{2}$, parabolically depending on the speed (Fig. 4). Fig. 5 shows the dependence of the maximum torque on the shaft $\left(M_{S}\right)_{\max }$ of the speed. Up to the speed $n^{*}$ a decrease of $\left(M_{S}\right)_{\max }=M_{0}+|\tilde{M}| \quad$ take place due to the compensation of the inertial forces components of the drive torque $B_{1} \sin 4 \omega t$. At speeds $n>n^{*}$ the torque $\left(M_{S}\right)_{\max }$ increases parabolically under the action of the inertial forces.

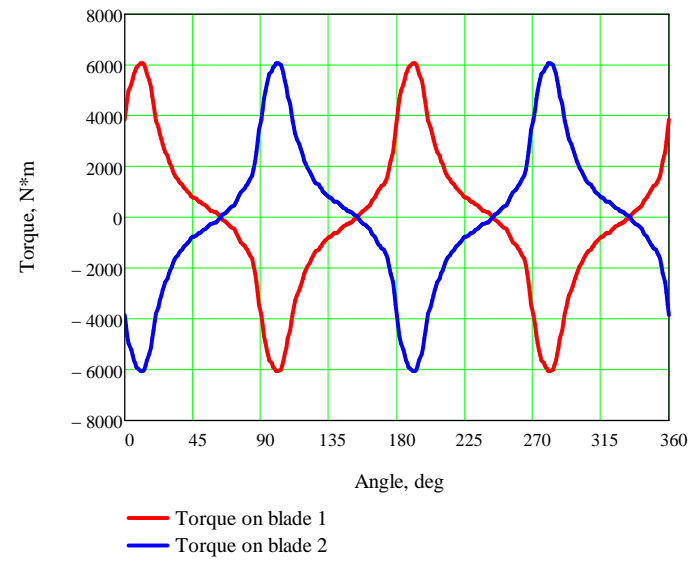

Fig. 2. Torques on the blades.

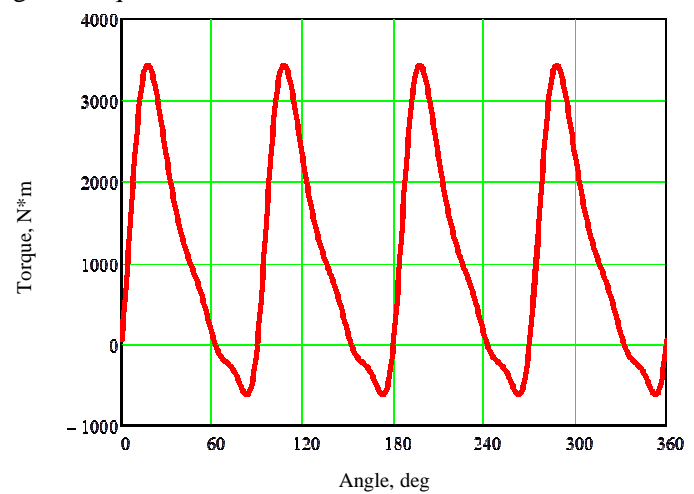

Fig. 3. The drive torque.

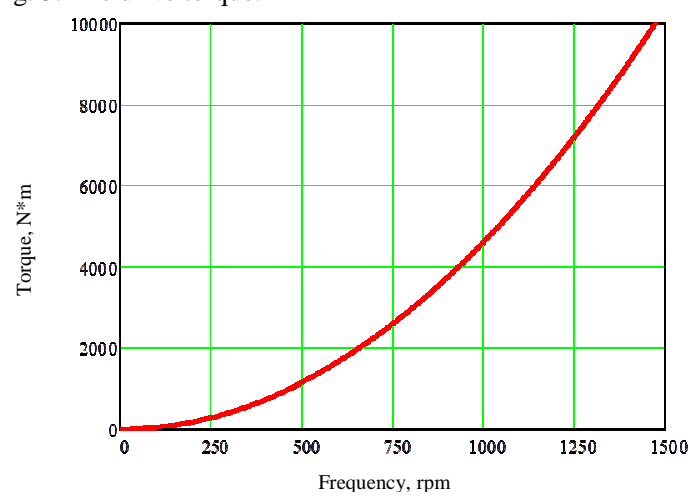

Fig. 4. Inertial moment

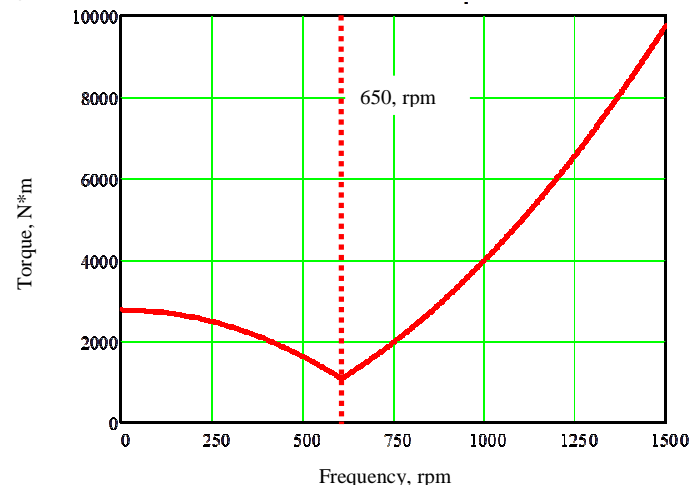

Fig. 5. Torque on the output shaft

\section{CONCLUSION}

In this article, we have obtained the analytical expressions for motor driving and inertial moments of the single-module rotary-vane engine with lever-cam referenced to the output shaft, also the condition under which the forces of inertia completely compensate the most significant component of the disturbing drive torque and minimizes the mechanical strength of the engine.

The results of numerical simulation obtained on the example of the engine with internal combustion, which are close to the theoretical prediction.

This results can be used in the preliminary design of the rotary-vane engines.

\section{REFERENCES}

[1] RF Patent 2374526 for an invention. Int. Cl. F16H25/04 Mechanism for converting motion / Lukyanov Y.N., Zhuravlev Y.N., Perminov A.L., Tikhonov S.I. et al. Publ. 27.11.2009. Bull. Number 33.

[2] RF Patent 2387844 for an invention. Int. Cl. F01G1/077, F02G1/044. Rotary-vane engine with an external supply of heat / Lukyanov Y.N., Zhuravlev Y.N., Perminov A.L., Tikhonov S.I. et al. Publ. 27.04.2010. Bull. Number 12.

[3] Zhuravlev Y.N., Lukyanov Y.N., Perminov A.L. Mathematical models of physical processes in the swingpiston engine with external heat supply. Collective monograph. Pskov state university. Pskov, 2012.

[4] Andreev M., Zhuravlev Y., Lukyanov Y., Perminov A. Autonomous Power Station Based on Rotary-Vane Engine with an External Supply of Heat. Environment. Technology. Resources. Proceedings of the 9th International Scientific and Practical Conference. Volume 2. 2013. 


\section{PRINCIPLES OF PUBLICATION USE AND ETHICS}

The Publisher states the following principles of publication use and ethics:

1. Only original works, that have not been previously published and in which the data do not infringe the copyright of another person or organization, are accepted for publishing.

2. Every submitted paper is reviewed anonymously by 2 reviewers.

3. Publisher has the right to use plagiarism detection software to screen submitted papers.

4. Authors retain copyright over their work, while allowing the conference to place this unpublished work under a Creative Commons Attribution License, which allows others to freely access, use.

5. Publisher undertakes to publish articles accepted after reviewing and, according to the scientific quality of an article, publish them electronically and include them in various international scientific paper data bases.

6. Publisher undertakes to use author's personal data only for the needs of identification of authors and co-authors, as well as to preserve data in accordance with the restrictions determined in the current regulations of the Republic of Latvia.

The author (-s) of original article confirms and guarantees that:

1. A paper has been written by the Author (s) who has (have) been stated in the Paper and in this statement and there is no any other person who would have any rights to this Paper.

2. The Paper is original; it has not been previously published and is notunder simultaneous consideration by another publications.

3. The Paper will notlater be submitted elsewhere.

4. There are references for all the pictures, data and other materials used in the Paper.

5. The results of the Paper are genuine; they do not infringe on the original work of another person or organisation. The Author (-s) have taken all the necessary measures to ensure the requirements of the sponsors' who finance the Paper.

6. If author finds a significant error after the publishing of the paper, it is the author's obligation to promptly notify the editor.

7. The Author (-s) has (have) been authorised to submit the Paper for publication; the intellectual property rights will not be infringed according to the 14th, 15th articles of the Copyright law and other normative acts of the Republic of Latvia and international normative acts binding for the Republic of Latvia.

8. The Author (-s) take (-s) the sole responsibility related to the third parties' objections and demands concerning the publishing or using the Paper.

9. The Author (s) take (-s) all the responsibility and promptly, irrevocably and unconditionally undertakes to independently at his (their) own expenses and risk if there will be objections or demands from the third parties' concerning the publishing or using the Paper.

\section{Publication editor:}

1. Ensures double-blind peer-review of the submitted paper.

2. Until the publishing, ensures the confidentiality of information concerning the submitted article.

3. Strives to prevent any possible conflict of interests between authors and reviewers or editors.

Reviewer:

1. Evaluates the content of the article according to the scientific article criteria, without regard to ethnic origin, gender, sexual orientation, citizenship, religious beliefs or political views of the authors.

2. Keeps confidential all the information related to submitted paper.

3. Notifies the editor if there is a suspicion of plagiarism or copyright infringement.

4. If the reviewer feels unqualified to review or knows that it is impossible to provide review in time, he should notify the Editor-in-Chief and ask to exempt him from reviewing. 
